\title{
A Portable Measurement Instrument for the Measurement of Water Body Chlorophyll-a in the Support of Fluorescence Detection
}

\author{
Cong Wang ${ }^{1}$, Daoliang $\mathrm{Li}^{1}$, Lingxian Zhang ${ }^{1, *}$, Qisheng Ding ${ }^{1,2}$, and Zetian $\mathrm{Fu}^{1}$ \\ ${ }^{1}$ College of Information and Electric Engineering, \\ China Agriculture University, Beijing, 100083, P.R. China \\ zlx131@163.com \\ ${ }^{2}$ School of Electrical Engineering and Automation, \\ Xuzhou Normal University, Xuzhou, 2211116, P.R. China
}

\begin{abstract}
Chlorophyll-a was regarded as the important indicator to describe the marine primary production because the chlorophyll a content of phytoplankton in the ocean is related to its photosynthesis production. The concentration of chlorophyll a is also the major parameter to evaluate marine water quality, organic pollution and detect the fishing ground, and the temporal and spatial variation of chlorophyll a contains the basic information of sea areas. Based on the spectral characteristics of chlorophyll fluorescence, this document recommends a new dual optical detecting instrument for the measurement of chlorophyll-a concentration, the microcontroller MSP430F149 as the key control module, by controlling the ultra-high brightness LED which wavelength is $450 \mathrm{~nm}$ to excite chlorophyll a to produce the fluorescent signal about $680 \mathrm{~nm}$, at the same time this LED is used as reference light, the dual-optical structure exclude the light fluctuations due to the impact of test results. At last, we get the relationship between relative fluorescence intensity and chlorophyll-a concentration with the spectrophotometer, we find the system has the good linear consistency when measures the low concentrations of chlorophyll-a.
\end{abstract}

Keywords: Chlorophyll-a, Relative fluorescence intensity, Dual-optical structure, Fluorescent signal.

\section{Introduction}

In recent years, China's Taihu, Wuhu lake, Songhua river and other water environment have emerged water crisis, due to the continued outbreak of blue-green algae classes in the water, making the phytoplankton in water sharply increase, the water oxygen exhaust, the water body is showing a large area of hypoxia and so large number of aquatic life is dying. As the rapid growth of algae, green algae accumulate on the

* Corresponding author. 
surface of the water, causing water problems, water body cannot exchange air with the outside world, day by day become foul, to the people's production, life and health have brought great harm.

The evaluation of eutrophication can be achieved by tracking and monitoring the chlorophyll content in water, all of which chlorophyll a is highest concentrations of chlorophyll, chlorophyll a determination can thus trace the lake's eutrophication $[1,2]$. In the mid-1970s, Soviet oceanographers first use pulse xenon lamp as the light source, narrow band interference filter and pulse detection technology for the development of pulsed underwater fluorometer. From the late 1970s to the early 1980s, many developed countries had been studying multi-parameter, multi-purpose drag fluorometer /conductivity, temperature, depth (CTD) sensor system development [3]. In 1997, K.Wild-Allen simultaneously measured water color and chlorophyll concentration by using the sea buoy quipped with a color sensor and fluorometer [4]. R. Barbini established laboratory of the fluorescent sensors which supplied for large-scale walking route monitoring from different conditions of seawater, monitoring object included the concentration of phytoplankton, yellow substance, turbidity, biomass productivity and organic pollutants [5]. In the late 1990s, TriOS companies (German) used super-efficient blue LEDs (wavelength 470nm) and the internal reference diode control energy output, selected photodiode as a monitor, successfully developed a portable microFlu-chl-type sensors which can be directly related to the different instrument to read continuous data and collect data, measurement range of $0 \sim 100 \mu \mathrm{g} / \mathrm{L}$, a resolution of $0.02 \mu \mathrm{g} / \mathrm{L}$, the accuracy for the optical reading of $3 \%$ (http://www.trios.de/). Such as these foreign chlorophyll fluorometer, there are many, but they are expensive, and their operation is more cumbersome. Laboratory methods of measuring the concentration of chlorophyll-a include high-performance liquid chromatography (HPLC) and spectrophotometry, they all need to extract chlorophyll from phytoplankton, not only the workload, and there is a lot of confounding factors in the whole process, the final result is likely to get the wrong data. Remote sensing methods need not extract, is an online measurement method, but only for a large area of water, and aerial work demanding, complex test results [6].

This paper presents a new site available for determination of chlorophyll fluorescence, high sensitivity, fast, real-time and good benefits, can be used for lakes, rivers, oceans and other water eutrophication on-site inspection $[7,8,9,10]$.

\section{Materials and Methods}

\subsection{Fluorescence Detection Principle}

When chlorophyll a is excited by the wavelength of 450nm light around, it will send around a wavelength of $680 \mathrm{~nm}$ fluorescence $[11,12,13]$, the fluorescence emission intensity:

$$
I_{f}=k Q I_{0}\left(1-e^{-\varepsilon c b}\right)
$$

Where: $k$ is the instrument constant; $Q$ is the fluorescence efficiency of material; $I_{0}$ is the light intensity of excitation source; $c$ is the concentration of substance; $b$ is the optical path difference of sample; $\varepsilon$ is the molar absorption coefficient. 
As long as the excitation light intensity is stable, the fluorescence intensity is only related with the concentration of chlorophyll a, chlorophyll-a concentration $\mathrm{c}$ and the relationship between the fluorescence intensity $\mathrm{F}$ :

$$
c=A-B \lg (D-F)
$$

Where: $A=2.3 \lg \left(k Q I_{0}\right) / \varepsilon b ; B=2.3 / \varepsilon b ; D=k Q I_{0}$. Therefore, the chlorophyll-a concentration can be detected by the detection of the received fluorescence intensity.

When the solution is very dilute, the total excitation energy is absorbed less than $5 \%$, or $\varepsilon c b \leq 0.05$, In Eq.(1), by power series expansion, since the second term is negligible, we get Eq.(3):

$$
I_{f}=k Q I_{0} \varepsilon c b
$$

In Eq.(3), when a fluorescent substance concentration is very dilute and the related excitation source is stable, fluorescence intensity is proportional to the concentration of the solution. However, when the concentration $c>0.05 / \varepsilon b$, the fluorescence intensity and concentration is not proportional, but when the concentration increases to a certain extent, to further increase the fluorescence intensity of concentration no longer enhanced. Because at this time, $e^{-\varepsilon c b} \approx 0$, so Eq. (1) becomes $I_{f}=k Q I_{0}$, At this point, no fluorescence intensity changes with concentration changes.

\subsection{Dual Beam and Dual-Channel Measuring}

By analyzing the Eq.(1), we can see, the changes in excitation light intensity has a great influence on the measurement results, so the system is designed with a dual optical path test structure(Fig.1). Two light paths are added interference filter and lens. By using the lens, the reference light and fluorescent light-gathering rate is enhanced, while the use of interference filter improves the conversion efficiency of photosensitive devices through filtering out most of the background light. In this System, photodiode converts the optical signal to light current, LEDs as the excitation light and reference light, its light intensity value is defined as $I_{0}$, and fluorescence signal intensity value is defined as $I_{f}$. Two signals processed through signal conditioning circuits are sent to the MSP430 microcontroller A/D side sampling (Fig.2), and finally by the microcontroller to calculate the ratio of two signals Eq.(4).

$$
I_{f} / I_{0}=k Q\left(1-e^{-\varepsilon c b}\right)
$$

For the dilute solution, we can get Eq.(5):

$$
I_{f} / I_{0}=k Q \varepsilon c b
$$




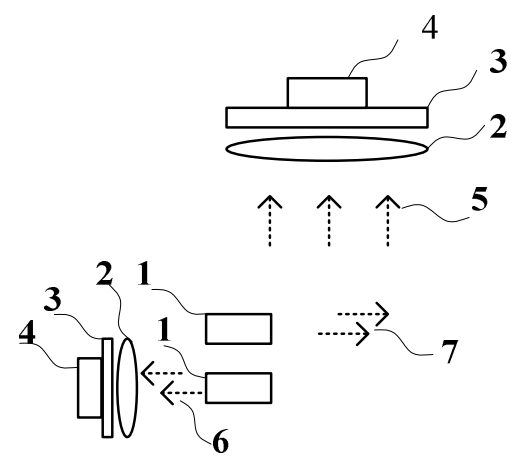

\section{Blue Leds 2.Convex Lens 3.Interference filter 4.Photodiode 5.Fluorometry 6. Reference light 7.Blue light}

Fig. 1. Fluorescence signal detection system

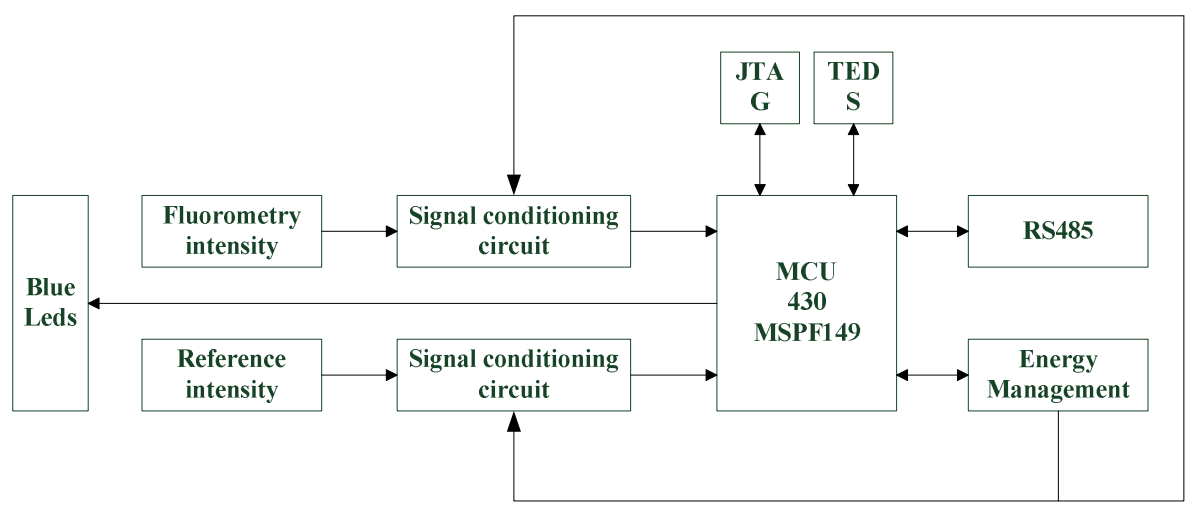

Fig. 2. Block diagram of the Chlorophyll a Intelligent instrument

Which can calculate the relative fluorescence intensity values to get the value of chlorophyll concentration. Therefore, the use of dual beam and dual-channel mode, by division and other operations to complete the normalization process, can effectively eliminate the excitation light intensity fluctuations on the measurement results, making the measurements more accurate. 


\subsection{Signal Conditioning Modules}

In Fig.1, fluorescence signal and reference signal via photosensitive device, the light signals into current signals, MSP430 MCU's internal analog-digital conversion chip cannot be directly on the current signal processing. System is involved in the signal conditioning circuitry, signal conditioning circuitry in addition to the current signal into a voltage signal, but also the role of a filter-amplifier, it can largely filter out the background and circuit noise from fluorescent signal and reference signal, and its amplification meets the MSP430 microcontroller chip sampling within the A/D requirements.

Fluorescence signal conditioning modules involving two amplifier circuit, completes the first stage current-voltage conversion, the conversion factor is denoted as $k_{1}$, also completes the second level voltage amplification, magnification is recorded as $A_{1}$. The reference light signal conditioning module is relatively simple, according to the reference light intensity with the strong characteristics, using only one amplifier, the current voltage conversion circuit, the conversion factor is denoted as $k_{2}$. We can calculate $U_{1}=I_{A} k_{1} A_{1}$ and $U_{2}=I_{B} k_{2}$, where: $I_{A}$ is light current from the fluorescence signal $; I_{B}$ is light current from the reference optical signal; $U_{1}$ is output voltage from the fluorescence signal conditioning circuit; $U_{2}$ is output voltage from the reference optical signal conditioning circuit.

In Fig.1, the two photodiode parameters are the same, so Eq.(6).

$$
I_{A} / I_{B}=I_{f} / I_{0}=U_{1} k_{2} / U_{2} k_{1} A_{1}=M U_{1} / U_{2}
$$

Where: $M=k_{2} / k_{1} A_{1}$.

The $M$ value can be regarded as a constant when calculate the Eq.(6).

\subsection{Microprocessor Module}

In order to reduce the energy consumption of the measuring device, this paper chooses TI's ultra low power MCU MSP430F149 as a core processor [14] (Fig.2), MSP430F149 embeds analog to digital conversion chip that can convert analog signals which come from signal conditioning module into digital signals, at the same time with the external power management module, microprocessor module also can supply energy for signal conditioning module. By using MSP430F149 internal timer A, we can control the duty cycle and frequency of the blue LEDs flashes, the system LEDs blinking frequency is $10 \mathrm{HZ}$, the ratio is 50\% [15]. MSP430F149 single-chip system via the JTAG interface to debug and download program. Moreover, in order to make the measurement devices more intelligent, able to achieve record and 
store the channel parameters and calibration parameters table, we design the spreadsheets (TEDS) in the flash of MSP430F149. The system uses RS485 bus output(Fig.2).

\subsection{Software Peak Detector Design}

In laboratory experiments, we detect the chlorophyll-a fluorescence signal waveform (Fig. 3).

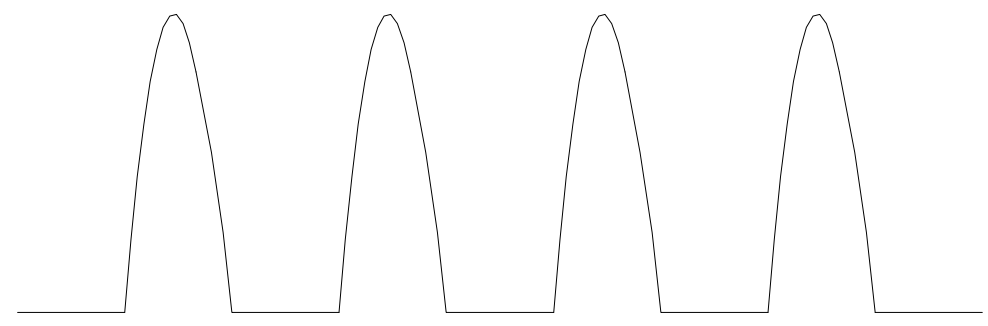

Fig. 3. Simulation of the Detection Waveform

This device which consists of interference filters, largely reduces the external impact of background light and emit blue light when obtains the fluorescence signal, and part of the design of the signal conditioning module blocking circuit, band pass filter and $50 \mathrm{HZ}$ notch filter, as the implementation of these circuits make the extraction of the fluorescent signal more pure. After analyzing of chlorophyll-a fluorescence signal waveform (Fig.3), the paper designs a software peak detection method, by controlling the microcontroller A/D converter chip sampling delay, to achieve in a half-cycle-interval sampled 255 times, whichever maximum value is stored. We define the average of the 10 consecutive maximum as the original voltage $U_{1}^{\prime}$ of the fluorescence signal, software peak detection method is also applicable to the original value of the reference signal acquisition, the original definition of the reference signal voltage is $U_{2}^{\prime}$. The final calculation of the relative fluorescence intensity can be expressed as $I_{f} / I_{0}=M U_{1} / U_{2}=M U_{1}^{\prime} / U_{2}^{\prime}=k Q\left(1-e^{-\varepsilon c b}\right)$ or $U_{1}^{\prime} / U_{2}^{\prime}=k Q\left(1-e^{-\varepsilon c b}\right) / M$, that is the results in theory which the system should get, so, we can see different concentrations of the measurement results should meet the negative exponential curve. Also we can get $U_{1}^{\prime} / U_{2}^{\prime}=k Q \varepsilon c b / M$ when the solution is very dilute, when, this should satisfy the linear time. Software flow chart shown as Fig.4. 


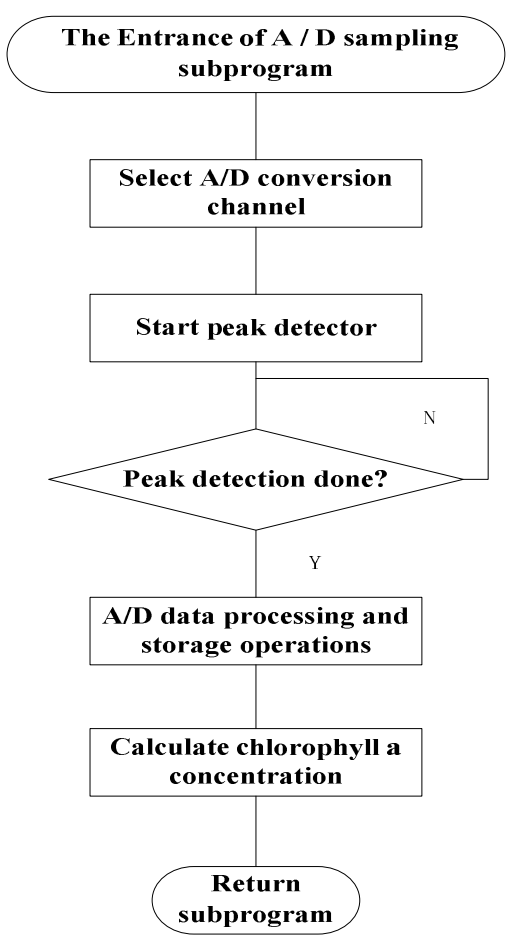

Fig. 4. A/D conversion module program

\section{Result and Discussion}

\subsection{Preparation of Chlorophyll-a Standard Solution}

In the preparation of chlorophyll a standard solution, we mainly extract chlorophyll-a by use of laboratory extraction method. First, select skeletonema costatum which was in the exponential phase of growth, weight class by its small to large is divided into A, $\mathrm{B}$ and $\mathrm{C}$, respectively, filter through a diameter of 0.45 acetate membrane, the membrane is then placed in $100 \mathrm{~mL}$ centrifuge tube, add $50 \mathrm{ml}$ of $90 \%$ concentration of acetone, the tube extraction at $4^{\circ} \mathrm{C}$ refrigerator 2 hours, then to $4000 \mathrm{r} / \mathrm{min}$ centrifugation for 5 minutes, last, collect the supernatant with $50 \mathrm{ml}$ brown flask in constant volume, and this solution as stock solution. When laboratory measurements, we can dilute the stock solution according to the needs of the required concentration. Through analysis of the nature of chlorophyll, we find the chemical properties of chlorophyll was unstable, so the prepared acetone solution of chlorophyll-a needs to be placed in dark place and the effective time for the solution is less 24 hours. 


\subsection{Evaluate of Instrument Performance and Standard Curve}

The chlorophyll fluorescence measuring instrument measuring range is $0 \sim 25 \mu \mathrm{g} / \mathrm{L}$, and the measuring minimum limit is $0.03 \mu \mathrm{g} / \mathrm{L}$. Instrument in actual measurement, the value of the relative fluorescence intensity is very small, not easy to device calibration and instrument performance analysis, so we select $U_{1}^{\prime} / U_{2}^{\prime}$ instead of $I_{f} / I_{0}$, the method has no effect on the calibration of instruments, and the rest of the photos or tables are processed in the same way. In the experiment of the instrument repeatability, we choose the prepared acetone solutions of the chlorophyll-a A and B as the experimental fluid, every $5 \mathrm{~min}$, respectively, measure repeatedly the two solutions five times, the measurement results as shown in Table 1. According to the definition of repeatability, we could calculate the standard deviation 0.0024 and 0.0021 by Bessel's formula [16], that proves the instrument has good repeatability.

Table 1. The data of repeatability experiment

\begin{tabular}{ccc}
\hline $\begin{array}{c}\text { The number of } \\
\text { measurements }\end{array}$ & $\begin{array}{r}\text { The relative fluorescence } \\
\text { intensity in acetone-A } \\
\text { solution }\end{array}$ & $\begin{array}{c}\text { The relative } \\
\text { fluorescence intensity } \\
\text { in acetone-B solution }\end{array}$ \\
\hline 1 & 1.422 & 2.114 \\
2 & 1.425 & 2.114 \\
3 & 1.421 & 2.111 \\
4 & 1.427 & 2.112 \\
5 & 1.425 & 2.109 \\
\hline
\end{tabular}

In the linearity test for the measuring instruments, we must first divide the concentration levels of acetone solution of chlorophyll-a. Delineation of specific: non-chlorophyll acetone solution is defined as the 0 level; after a 1/8 fold dilution of the acetone solution of chlorophyll $\mathrm{A}$ is defined as 1/8 level; after 1/4 times the concentration of chlorophyll in acetone diluted solution $\mathrm{A}$ is defined as $1 / 4$ level; after $1 / 2$ times the concentration of chlorophyll in acetone diluted solution $\mathrm{A}$ is defined as $1 / 2$ level; the original acetone solution of chlorophyll $\mathrm{A}$ is defined as 1 level; original chlorophyll acetone solution B is defined as Level 2 and the original chlorophyll acetone solution $\mathrm{C}$ is defined as the 3 level. The relationship between the average of relative fluorescence intensity from experimental measurement and chlorophyll concentration levels can be described as in Fig.5.

Fig. 5 shows the measured relative fluorescence intensity close to the negative index, and theoretical analysis of the relative fluorescence intensity: $I_{f} / I_{0}=k Q\left(1-e^{-\varepsilon c b}\right)$ is very close to this. It can be seen, if the concentration increased to a certain extent, the fluorescence intensity will not change significantly. 


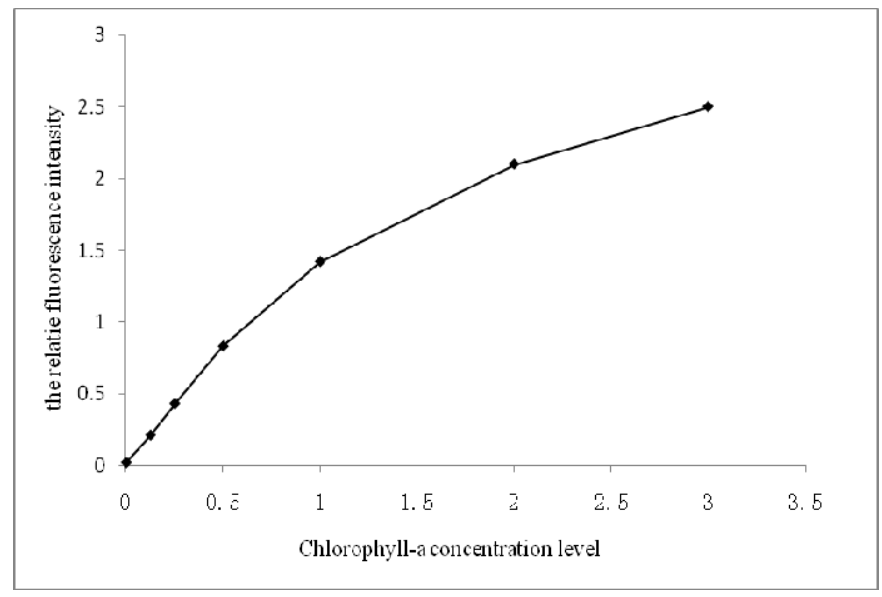

Fig. 5. Detecting results of the relative fluorescence intensity

In order to analyze the relationship between the relative fluorescence intensity and concentration, we has measured $0,1 / 8,1 / 4,1 / 2$ and 1 grade acetone concentration of chlorophyll-a for getting the value of the relative fluorescence intensity. In theory, the relationship between the concentration of the diluted solution and the relative fluorescence intensity is $I_{f} / I_{0}=k Q \varepsilon c b$. In Fig.6, the relative fluorescence intensity and concentration is in a linear relationship, which meets the theoretical results of the analysis, so the system design is feasible.

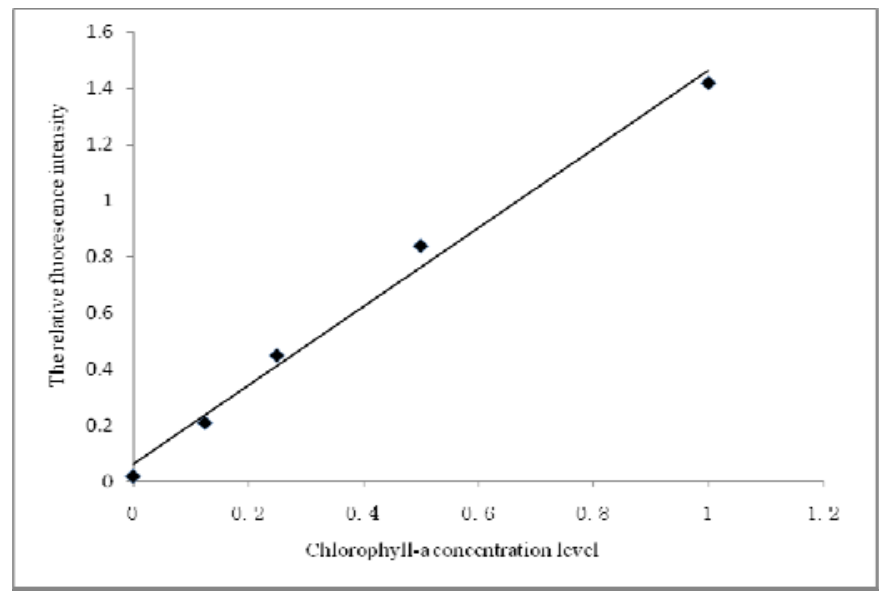

Fig. 6. Detecting results of the relative fluorescence intensity in weak acetone solution

According to national standard methods, we detect the concentration of the five levels of chlorophyll dilute solution with multi-wavelength spectrophotometer method [17], the measuring results is $0 \mu \mathrm{g} / \mathrm{L}, 3.05 \mu \mathrm{g} / \mathrm{L}, 6.32 \mu \mathrm{g} / \mathrm{L}, 12.53 \mu \mathrm{g} / \mathrm{L}$ and $19.82 \mu \mathrm{g} / \mathrm{L}$ from small 
to large order. As the Fig.7 shown, fluorescence intensity converted into concentration by the equation:

$$
y=14.2 x-0.01
$$

Where: $x$ is the relative fluorescence intensity; $y$ is the chlorophyll-a concentration.

The linear regression coefficient is 0.997 , that is, the measuring device in case of low value has a good linear consistency.

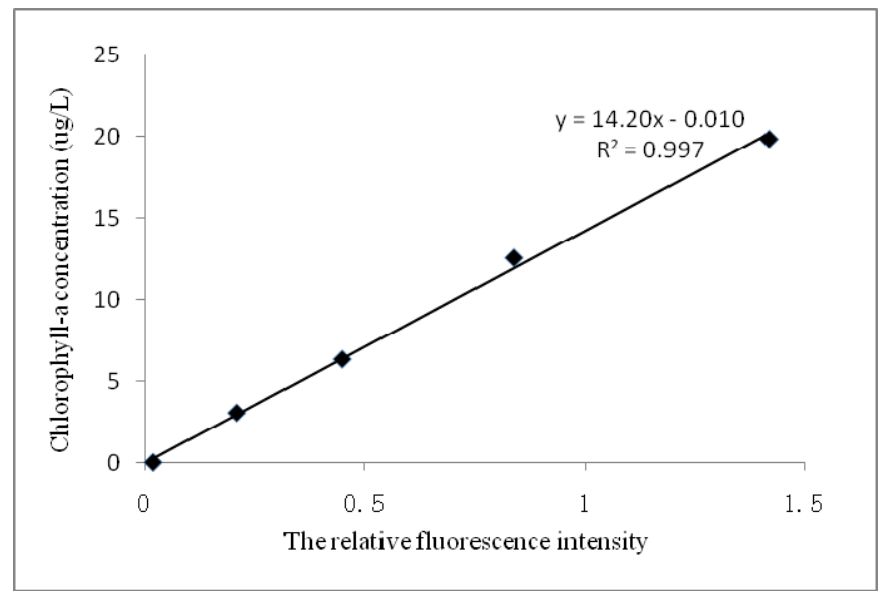

Fig. 7. Testing results using spectrophotometer

\section{Conclusion}

In this article, we carefully study the fluorescence detection method for measuring the chlorophyll-a content and chlorophyll-a fluorescence characteristics, combined with domestic and international issues related to research, design a low-power, low noise, high speed chlorophyll-a content measuring device.

(1) The system chooses MSP430F149 microcontroller as the core of the detection control unit, its rich pin function and the built-in functional unit simplify the system's design, while improve the system's stability and anti-jamming capability.

The system uses ultra-high brightness, a wavelength of about 460nm blue LEDs as (2) the excitation source, while the use of single-chip PWM output control, and other functional units select low-power devices for designing, effectively reduce the system power consumption, system overall average power consumption is only $0.03 \mathrm{~W}$.

(3) Due to the light fluctuations, test results may not be accurate, so, in order to exclude the impact, the system applies the structure of dual optical detection. When debugging is complete, we select different concentrations of chlorophyll acetone solution for measurement, analysis the relationship between the relative fluorescence intensity and concentrations of chlorophyll-a, and then complete the calibration 
solution of chlorophyll with the spectrophotometer, the system repeatability tests and linearity tests. As can be seen from the experimental data, the system has the good linear consistency when measures the low concentrations of chlorophyll-a.

Acknowledgements. This work was supported by the programs "Development and Applications of sensor network applied to monitor bloom of blue-green algae in Taihu Lake" (2010ZX03006-006), and Beijing Natural Science Foundation "Integrations methods of digitalization technologies in intensive fish farming" (4092024).

\section{References}

1. Daying, X., Zhenxian, W., Jingfang, X.: Study on in-situ measuring set for chlorophyll-a in the seawater. Oceanologia Et Limnologia Sinica 20(4), 472 (2003)

2. Mingcui, W., Xueqin, L., Jianhui, Z.: Evaluation method and classification standard on lake eutrophication. Environmental Monitoring in China 18(5), 47 (2002)

3. Ruth, B.: A Device for the Determination of the Microsecond Component of the in vivo Chlorophyll Fluorescence Induction Kinetics. Meas. Sci. Technol. (1), 517 (1990)

4. Wild-Allen, K., Tett, P., Bowers, D.: Observations of diffuse upwelling irradiance and chlorophyll in case I waters near the Canary Islands (Spain). Optics \& Laser Technology 29(1), 3 (1997)

5. Barbini, R., Colao, F., Fantoni, R., et al.: Fluorescence Determination of Pollutants and Natural Components in Seawater from the on Board Mobile Lidar Apparatus. SPIE (3534), 359 (1998)

6. Yunwu, L., Shuzhi, Z., Yunguo, G.: Satellite remote sensing in the ocean observing application. Aerospace China (8), 3 (1997)

7. Beutler, M.: A fluorometric method for the differentiation of algal populations in vivo and situ. Photosynthesis Research 72, 39 (2002)

8. Zhigang, W.: The phytoplankton classified measure based on excitation fluorescence spectra technique. China Environmental Science 28(3), 329 (2008)

9. Lianhua, C., Lei, L.: Application of the chlorophyll fluorescence in photosynthesis of alga. Jiangxi Science 25(6), 788 (2007)

10. Sharma, S., Schulman, S.G.: Introduction to fluorescence spectroscopy, pp. 22-23. John Wiley and Sons. Inc., New York (1999)

11. Ruth, B.: A Device for the Determination of the Microsecond Component of the in vivo Chlorophyll Fluorescence Induction Kinetics. Meas. Sci. Technol. (1), 517 (1990)

12. Guozhen, C.: Fluorescence analysis, p. 12. Science Press, Beijing (1975)

13. Brechet, E., Stay, D.M., Wakefield, R.D., et al.: A novel blue LED based scanning hand-held fluorometer for detection of terrestrial algae on solid surfaces. SPIE 3414, 184 (1998)

14. Lierda.MSP430F13X/F14X Chinese data sheet [EB / OL] (2005), http: / / www.1ierda.com

15. Jian, Q., Guanling, Y., Zhenjiang, H., et al.: Atmospheric S02 concentration analyzer based on ultra-violet fluorescence. Chinese Journal of Scientific Instrument 29(1), 175 (2008)

16. Taylor, J.R.: An Introduction to Error Analysis, pp. 294-298. University Science Books (1981)

17. State Environmental Protection Administration 《Water and wastewater monitoring and analysis methods $\gg$ Editorial Board. Water and wastewater monitoring and analysis methods, 4th edn., pp. 670-671. China Environmental Science Press, Beijing (2002) 